\title{
Analysis of Motor Complication and Relative Factors in a Cohort of Chinese Patients with Parkinson's Disease
}

\author{
Baihua Sun $\left(\mathbb{D},{ }^{1}\right.$ Tao Wang, ${ }^{2}$ Nianying Li, ${ }^{1}$ and Jin Qiao $\mathbb{D}^{1,3}$ \\ ${ }^{1}$ Department of Neurology, The First Affiliated Hospital of Xi'an Jiaotong University, Xi'an 710061, China \\ ${ }^{2}$ Department of Neurology, The Sengong Hospital, Xi'an 710300, China \\ ${ }^{3}$ Department of Rehabilitation Medicine, The First Affiliated Hospital of Xi'an Jiaotong University, Xi'an 710061, China
}

Correspondence should be addressed to Jin Qiao; qiaojn123@163.com

Received 7 July 2019; Revised 9 April 2020; Accepted 7 July 2020; Published 29 July 2020

Academic Editor: Fabrizio Stocchi

Copyright ( 92020 Baihua Sun et al. This is an open access article distributed under the Creative Commons Attribution License, which permits unrestricted use, distribution, and reproduction in any medium, provided the original work is properly cited.

Objective. Motor complications are common in Parkinson's disease (PD). The reported occurrence of motor complications varies across regions and races. The aim of our study was to describe the development of dyskinesias and motor fluctuations among Chinese PD patients and the relative risk factors. Methods. In the current cross-sectional survey study, PD patients with motor fluctuations and dyskinesia were enrolled from March to November 2018 in Shaanxi province, a northwest area of China. Data were collected by the movement disorder specialists. A self-designed questionnaire was utilized during face-to-face interviews. In addition, the relevant factors of motor complications were analyzed by univariable and multivariable analyses. Results. Of the166 PD patients recruited, $52(31.33 \%)$ and $25(15.06 \%)$ patients had motor fluctuations and dyskinesia, respectively, which occurred in $6.76 \pm 3.77$ and $8.61 \pm 4.46$ years after the onset of motor symptoms and $5.37 \pm 3.33$ and $6.80 \pm 3.43$ years after the treatment of levodopa therapy, respectively. Patients with motor fluctuations and dyskinesias had longer disease duration, younger onset age, higher Hoehn-Yahr stages and UPDRS III scores, higher daily levodopa dosage and levodopa equivalent daily dose (LEDD), and longer duration of levodopa treatment $(P<0.05)$. Bradykinesia-rigidity dominant patients had higher incidences of motor fluctuations $(61.54 \%$ vs $38.46 \%)$ and dyskinesias $(68.00 \%$ vs $32.00 \%)$ than tremor-dominant patients $(P<0.05)$. Results of the multivariate logistic regression analyses showed that the duration of levodopa therapy, age of the onset, and bradykinesia-rigidity dominant type were independent risk factors of motor fluctuations $(P<0.05)$. In addition, duration of disease and bradykinesiarigidity dominant type were independent risk factors of dyskinesia $(P<0.05)$. Conclusions. The rate of motor fluctuations was higher than dyskinesias in Chinese patients with Parkinson's disease. Patients with younger age onset, bradykinesia-rigidity dominant type, longer disease duration, and longer duration of levodopa therapy are more likely to develop motor complications.

\section{Introduction}

Parkinson's disease (PD) is the second most prevalent neurodegenerative disorder of the central nervous system. Dopamine replacement therapy, which is considered as one of the main treatment methods, may lead to severe untoward effects, such as motor fluctuations and dyskinesias. Previous studies have indicated that approximately $40 \%$ PD patients who has been treated with levodopa for 4-6 years experienced wear-off (WO) and levodopa-induced dyskinesia [1], and $74 \%$ patients developed at least one motor complications within 4 years after starting treatment with levodopa $[2,3]$. However, the incidence of motor complications is different in Asian countries. A Japanese investigation study showed that the percentage of patients with developed WO was $21.3 \%, 59.4 \%$, and $73.2 \%$ by the end of the $5^{\text {th }}, 10^{\text {th }}$, and $15^{\text {th }}$ year after disease onset, respectively [4]. In a Chinese multicenter registry survey, the incidence rates of $\mathrm{WO}$ and dyskinesia were $46.5 \%$ and $10.3 \%$, which were lower than that in Western countries [5]. During the "on/off" periods, patients may experience fluctuations in motor and nonmotor symptoms. Another study reported that approximately 50\% subjects may develop motor or nonmotor fluctuation within 5 years after levodopa therapy [6]. The etiology of motor complications is not well understood. Some studies have found that the patients with younger age 
of onset, longer duration of disease, higher daily levodopa dosage, severity of disease, and longer duration of levodopa therapy are more likely to develop motor complications. Few studies have shown that women, bradykinesia-dominant type, and low body weight are risk factors of dyskinesia [6]. Therefore, prevalence of motor complications and relative factors in PD is still controversial. In addition to the disease itself, treatment with levodopa is an important factor for the developing of motor fluctuations. New insight into motor complications of this disabled disease is urgently needed since they can be temporarily controlled but not fully eradicated. Therefore, it is crucial to clarify the potential risk factors for motor fluctuations and dyskinesia in different populations. The aim of the present study was to describe the development of dyskinesias and motor fluctuations among the PD patients, and related risk factors were also investigated.

\section{Materials and Methods}

2.1. Subjects and Basic Data. A cross-sectional study was performed on consecutive patients with PD. All patients were recruited from the First Affiliated Hospital of Xi'an Jiaotong University from March to November 2018 in Shaanxi province, a northwest area of China. All the participants were diagnosed according to Movement Disorders Society (MDS) criteria for Parkinson's disease [7]. Patients with severe cognitive dysfunction who could not complete study' rating scales were excluded. A self-designed questionnaire was used to ascertain the patients' information including gender, age, body massing index (BMI), disease duration, onset age, clinical types, and medications. Levodopa equivalent daily dose (LEDD) was calculated according to previous report [8].

\subsection{Clinical Evaluation of Motor Complications and Clinic} Characteristics. The presence of motor fluctuations included wearing-off (WO) and on-off phenomenon; WO was defined as "the generally predictable recurrence of motor and nonmotor symptoms before the scheduled doses of antiparkinsonian medication and improvements after next doses [9-11]." "On-off phenomenon" is characterized by ongoing predictable or unpredictable switching from being "on" with mobile with dyskinesia to being "off" and immobile during the course of a day [12]. Dyskinesia was defined as an involuntary, purposeless, predominantly choreic movement involving the muscles of the limbs, neck, trunk, or rarely face during the "on" period of PD patients [2]. Freezing of gait (FOG) is defined as "an episodic inability (lasting seconds) to generate effective stepping in the absence of any known cause other than parkinsonism or high-level gait disorders. It is most commonly experienced not only during turning and step initiation but also when faced with spatial constraint, stress, and distraction. Focused attention and external stimuli (cues) can overcome the episode [13]. " Clinical assessments for motor fluctuations and dyskinesia phenomenon were performed in all patients. The severity of motor symptoms was recorded using the Unified
Parkinson's Disease Scale part III (UPDRS III), and the PD disease severity was quantified using the modified Hoehn-Yahr stage. The tremor score was defined as the sum of UPDRS part II item 16 (self-report), part III item 20 (resting tremor), and part III item 21 (action/positional tremor). The nontremor score was defined as the sum of UPDRS part II items 5, 7, 12, and 15 and part III items 18, 19, 22 , and 31 . According to prevalent motor symptoms, subjects were divided into two subtypes: tremor predominant type (tremor score/nontremor score $>1$ ) and bradykinesia-rigidity predominant type (tremor score/nontremor score $\leq 1$ ).

All the enrolled patients agreed to participate in the study and gave full consent as dictated by the First Affiliated Hospital of Xi'an Jiaotong University ethics committee. All the assessments were performed by movement disorders specialists during face-to-face interviews with the patients.

2.3. Statistical Analysis. Statistical analysis was performed with SPSS version 24 (IBM). Normality of data for each individual parameter was assessed using the Schapiro-Wilk test. The differences in the clinical and demographic characteristics of the patients with and without motor complications and continuous variables, if normally distributed, were presented as mean $\pm S D$, and two groups were compared by the 2 -tailed $t$-test. If not normally distributed, continuous variables were presented as median (quartile) and compared by the nonparametric test. Discrete variables were compared by the chi-square test. A multivariate logistic regression analysis was performed. Factors with $P<0.1$ were included in a multivariate logistic regression analysis to determine the independent risk factors for motor fluctuations and dyskinesia. $P<0.05$ was considered statistically significant.

\section{Results}

3.1. Patients Characteristics. One hundred and sixty-six patients with PD including 89 men and 77 women, with a mean age of $65.80 \pm 9.11$ years, mean disease duration of $4.96 \pm 3.90$ years, and mean age of $60.83 \pm 9.91$ years at onset of disease, were enrolled in the study. Table 1 summarizes their demographic data and clinical characteristics. According to the modified Hoehn-Yahr stage, 18 patients $(10.84 \%)$ were in stage 1,33 patients $(19.88 \%)$ were in stage $1.5,56(33.73 \%)$ patients were in stage 2, 20 patients (12.05\%) were in stage $2.5,27$ patients $(16.27 \%)$ were in stage 3 , and 12 patients $(7.23 \%)$ were in stage 4 . Eighty-nine patients (53.61\%) were tremor-dominant types, and 77 patients (46.39\%) were bradykinesia-rigidity types. The daily levodopa dosage was $468.87 \pm 267.41 \mathrm{mg} / \mathrm{d}$, and the duration of levodopa therapy was $2.93 \pm 3.67$ years.

Among them, 135 patients $(81.33 \%)$ were treated with levodopa, 92 patients (55.42\%) with dopamine agonists, 44 patients $(26.51 \%)$ with monoamine oxidase B (MAO-B) inhibitor, 9 patients $(5.42 \%)$ with catechol-oxyl-methyltransferase (COMT) inhibitor, 26 patients (15.66\%) with anticholinergic drug, and 16 patients (9.64\%) with amantadine. 
TABle 1: Demographic, clinical, and medical characteristics for our subjects.

\begin{tabular}{|c|c|}
\hline Characteristics & Value \\
\hline Gender (male/female) & $89 / 77$ \\
\hline Age (years, mean $\pm S D)$ & $65.8 \pm 9.11$ \\
\hline Onset age (years, mean $\pm S D)$ & $60.83 \pm 9.91$ \\
\hline Disease duration (years, mean $\pm S D$ ) & $4.96 \pm 3.90$ \\
\hline \multicolumn{2}{|l|}{ Clinical phenotypes $(n, \%)$} \\
\hline Tremor & $89(53.61)$ \\
\hline Bradykinesia-rigidity & $77(46.39)$ \\
\hline \multicolumn{2}{|l|}{ Modified Hoehn-Yahr stages $(n, \%)$} \\
\hline 1 & $18(10.84)$ \\
\hline 1.5 & $33(19.88)$ \\
\hline 2 & $56(33.73)$ \\
\hline 2.5 & $20(12.05)$ \\
\hline 3 & $27(16.27)$ \\
\hline 4 & $12(7.23)$ \\
\hline UPDRS scores $($ mean $\pm S D)$ & $39.93 \pm 18.80$ \\
\hline UPDRS III scores (mean \pm SD) & $22.19 \pm 11.83$ \\
\hline Daily levodopa dosages (mg) & $474.20 \pm 268.76$ \\
\hline $\mathrm{LEDD}(\mathrm{mg} / \mathrm{d}$, mean $\pm \mathrm{SD})$ & $457.48 \pm 386.00$ \\
\hline L-dopa duration (years, mean $\pm S D$ ) & $2.93 \pm 3.68$ \\
\hline \multicolumn{2}{|l|}{ Medications $(n, \%)$} \\
\hline Levodopa-benserazide & $135(81.33)$ \\
\hline Levodopa-carbidopa & $11(6.63)$ \\
\hline Dopamine agonist & $92(55.42)$ \\
\hline MAO-B inhibitor & $44(26.51)$ \\
\hline COMT inhibitor & $9(5.42)$ \\
\hline Anticholinergic & $26(15.66)$ \\
\hline Amantadine & $16(9.64)$ \\
\hline
\end{tabular}

Abbreviations: SD, standard deviation; UPDRS: unified Parkinson disease rating scale; LEDD: levodopa equivalent daily dose; MAO: monoamine oxidase; and COMT: catechol-o-methyltransferase.

3.2. The Characteristics and Prevalence Rates of PD Patients with Motor Fluctuations and Dyskinesia. Of all patients, 52 patients (31.33\%) and 25 patients $(15.06 \%)$ had symptoms of motor fluctuations and dyskinesia, respectively, which occurred $6.76 \pm 3.77$ and $8.61 \pm 4.46$ years after the onset of motor symptoms and $5.37 \pm 3.33$ and $6.80 \pm 3.43$ years after levodopa therapy, respectively. Additionally, 10 patients (6.02\%) experienced freezing of gait.

Among patients with motor fluctuations, 46 patients (88.46\%) experienced WO, and 6 patients (11.54\%) experienced on-off phenomenon, Among patients with dyskinesia, 17 patients (68.0\%) experienced peak-dose dyskinesia, 3 patients $(12.0 \%)$ experienced biphasic dyskinesia, and 5 patients $(20.0 \%)$ experienced dystonia.

3.3. The Relative Factors of PD Patients with Motor Fluctuations Based on Univariate Analysis. In comparison with patients without motor fluctuations, patients with motor fluctuations had older age, younger onset age, longer disease duration, more severely modified Hoehn-Yahr stage and UPDRS III; score, higher daily levodopa dosage and levodopa equivalent daily dose (LEDD), and longer duration of levodopa treatment $(P<0.05)$. In patients with motor fluctuations, the proportion of levodopa-benserazide, levodopa-carbidopa, dopaminergic agonists, COMT inhibitors, and amantadine was higher than that in patients without motor fluctuation $(P<0.05)$. Patients with bradykinesia-rigidity were more prone to motor fluctuations $(P<0.05)$. The specific results are shown in Table 2.

\subsection{The Relative Factors of PD Patients with Dyskinesia Based} on Univariate Analysis. In comparison with patients without motor complications, patients with motor dyskinesia had age older, longer disease duration, younger onset age, more severely modified Hoehn-Yahr stage and UPDRS III score, higher daily levodopa dosage and levodopa equivalent daily dose (LEDD), and longer duration of levodopa treatment $(P<0.05)$. In patients with dyskinesia, the proportion of levodopa-benserazide, levodopa-carbidopa, dopaminergic agonists, and COMT inhibitors was higher than that in patients with motor complications $(P<0.05)$. Patients with bradykinesia-rigidity were more prone to dyskinesia $(P<0.05)$. The specific results are shown in Table 3.

3.5. The Results of Motor Fluctuations and Dyskinesia in Different Clinical Stages. PD patients with younger age at onset tended to develop more motor fluctuations and dyskinesia, especially when under 50 years old. The incidence of motor fluctuations and dyskinesia were $62.07 \%$ and $31.03 \%$, which occurred at $3.62 \pm 4.29$ and $2.54 \pm 4.40$ years of levodopa therapy. Motor complications increased with the duration of the disease and duration of L-dopa treatment. The dose of levodopa therapy was also related to the incidence of motor complication. PD patients taking higher daily doses, particularly over $600 \mathrm{mg} /$ day, compared with lower doses of levodopa increased the risk of motor complications $(P<0.05)$. The specific results are presented in Table 4.

3.6. The Relative Factors of PD Patients with Dyskinesia Based on Multivariate Analysis. The results of multivariate logistic regression analyses showed that the duration of levodopa therapy, age of onset, and bradykinesia-rigidity dominant type were independent risk factors of motor fluctuations. In addition, duration of disease and bradykinesia-rigidity dominant type were independent risk factors of dyskinesia. The onset age was related to the risk of developing motor fluctuations $(\mathrm{OR}=0.933,95 \% \quad \mathrm{CI}: \quad 0.888 \sim 0.980)$; the younger onset was positive related with the risk of motor fluctuations. In addition, the clinical phenotype of PD was associated with the occurrence of motor complications. The occurrence of motor fluctuations in bradykinesia-rigidity dominant type was three times higher than that in tremordominant type (OR $=3.051,95 \% \mathrm{CI}: 1.164 \sim 7.997)$, and the occurrence of dyskinesia in bradykinesia-rigidity dominant type was eight times higher than that in tremor-dominant $(\mathrm{OR}=8.170,95 \% \mathrm{CI}: 1.862 \sim 35.849)$. Patients with longer duration of levodopa therapy were more likely to develop WO $(\mathrm{OR}=1.030,95 \% \mathrm{CI}: 1.061 \sim 1.043)$. The longer the duration of disease was, the greater the risk of dyskinesia was $(\mathrm{OR}=1.359,95 \% \mathrm{CI}: 1.161 \sim 1.590)$. The specific results are shown in Table 5. 
TABLE 2: Characteristics of PD patients with or without motor fluctuations.

\begin{tabular}{|c|c|c|c|c|}
\hline Motor fluctuations & With $(n=52)$ & Without $(n=114)$ & $t / \chi^{2}$ & $P$ value \\
\hline Sex (male/female) & $29 / 23$ & $60 / 54$ & 0.141 & 0.707 \\
\hline Age $($ mean $\pm \mathrm{SD}, y)$ & $63.55 \pm 9.30$ & $66.92 \pm 8.86$ & 2.215 & 0.029 \\
\hline Onset age $($ mean $\pm \mathrm{SD}, y)$ & $55.33 \pm 9.81$ & $63.41 \pm 8.93$ & 5.232 & $<0.001$ \\
\hline Disease duration (median, quartile, $y$ ) & $7(5 \sim 11)$ & $3(2 \sim 5)$ & 7.293 & $<0.001$ \\
\hline Clinical phenotypes $(n, \%)$ & & & 6.991 & 0.008 \\
\hline Tremor & $20(38.46)$ & $69(60.52)$ & & \\
\hline Bradykinesia-rigidity & $32(61.54)$ & $45(39.47)$ & & \\
\hline Modified Hoehn-Yahr stages (median, quartile) & $2.5(2 \sim 3)$ & $2(1.5 \sim 2.5)$ & 4.770 & $<0.001$ \\
\hline UPDRS III scores (mean $\pm \mathrm{SD}$ ) & $25.71 \pm 13.93$ & $20.59 \pm 10.43$ & 2.358 & 0.021 \\
\hline Daily levodopa dosage (mg, median, quartile) & $500(375 \sim 750)$ & $375(375 \sim 425)$ & 3.353 & 0.001 \\
\hline $\mathrm{LEDD}(\mathrm{mg} / \mathrm{d}$, mean $\pm \mathrm{SD})$ & $670.07 \pm 411.89$ & $356.06 \pm 329.37$ & 6.475 & $<0.001$ \\
\hline L-dopa duration $($ mean $\pm \mathrm{SD}, y)$ & $6.06 \pm 3.98$ & $1.52 \pm 2.46$ & 6.825 & $<0.001$ \\
\hline \multicolumn{5}{|l|}{ Medications $(n, \%)$} \\
\hline Levodopa-benserazide & $50(96.15)$ & $85(74.56)$ & 10.963 & 0.001 \\
\hline Levodopa-carbidopa & $8(15.38)$ & $3(2.63)$ & 9.387 & 0.002 \\
\hline Dopamine agonist & $38(73.08)$ & $54(47.37)$ & 9.553 & 0.002 \\
\hline MAO-B inhibitor & $17(32.69)$ & $27(23.68)$ & 1.488 & 0.223 \\
\hline COMT inhibitor & $9(17.31)$ & $0(0.00)$ & 20.862 & $<0.001$ \\
\hline Anticholinergic & $7(13.46)$ & $19(16.67)$ & 0.278 & 0.598 \\
\hline Amantadine & $9(17.31)$ & $7(6.14)$ & 5.113 & 0.024 \\
\hline
\end{tabular}

Abbreviations: SD, standard deviation; UPDRS: unified Parkinson disease rating scale; LEDD: levodopa equivalent daily dose; Mao: monoamine oxidase; and COMT: catechol-o-methyltransferase.

TABle 3: Characteristics of PD patients with or without dyskinesia.

\begin{tabular}{|c|c|c|c|c|}
\hline Dyskinesia & With $(n=25)$ & Without $(n=141)$ & $t / \chi^{2}$ & $P$ value \\
\hline Sex (male/female) & $16 / 9$ & $73 / 68$ & 1.227 & 0.259 \\
\hline Age $($ mean $\pm \mathrm{SD}, y)$ & $65.92 \pm 10.08$ & $65.84 \pm 8.97$ & 0.042 & 0.966 \\
\hline Onset age $($ mean $\pm \mathrm{SD}, y)$ & $55.92 \pm 11.18$ & $61.74 \pm 9.46$ & 2.755 & 0.007 \\
\hline Disease duration (median, quartile, $y$ ) & $9(6 \sim 13.5)$ & $3(2 \sim 5)$ & 6.113 & $<0.001$ \\
\hline Clinical phenotypes $(n, \%)$ & & & 5.529 & 0.019 \\
\hline Tremor & $8(32.00)$ & $81(57.45)$ & & \\
\hline Bradykinesia-rigidity & $17(68.00)$ & $60(42.55)$ & & \\
\hline Modified Hoehn-Yahr stages (median, quartile) & $3(2.75 \sim 3.5)$ & $2(1.5 \sim 2.5)$ & 5.569 & $<0.001$ \\
\hline UPDRS III scores (mean \pm SD) & $33.08 \pm 15.18$ & $20.26 \pm 10.03$ & 4.065 & $<0.001$ \\
\hline Daily levodopa dosage (mg, median, quartile) & $500(375 \sim 750)$ & $375(375 \sim 500)$ & 2.175 & 0.030 \\
\hline $\operatorname{LEDD}(\mathrm{mg} / \mathrm{d}$, mean $\pm \mathrm{SD})$ & $796.15 \pm 544.42$ & $395.22 \pm 314.25$ & 4.723 & $<0.001$ \\
\hline L-dopa duration $($ mean $\pm \mathrm{SD}, y)$ & $7.40 \pm 3.81$ & $2.15 \pm 3.04$ & 5.481 & $<0.001$ \\
\hline \multicolumn{5}{|l|}{ Medications $(n \%)$} \\
\hline Levodopa-benserazide & $24(96.00)$ & $111(78.72)$ & 4.173 & 0.041 \\
\hline Levodopa-carbidopa & $7(28.00)$ & $4(2.84)$ & 21.731 & $<0.001$ \\
\hline Dopamine agonist & $19(76.00)$ & $73(51.77)$ & 5.405 & 0.025 \\
\hline MAO-B inhibitor & $9(36.00)$ & $35(24.82)$ & 1.365 & 0.243 \\
\hline COMT inhibitor & $5(20.00)$ & $4(2.84)$ & 12.199 & $<0.001$ \\
\hline Anticholinergic & $6(24.00)$ & $24(17.02)$ & 0.698 & 0.403 \\
\hline Amantadine & $2(8.00)$ & $10(7.02)$ & 0.026 & 0.087 \\
\hline
\end{tabular}

Abbreviations: SD, standard deviation; UPDRS: unified Parkinson disease rating scale; LEDD: levodopa equivalent daily dose; Mao: monoamine oxidase; and COMT: catechol-o-methyltransferase.

\section{Discussion}

Motor complications are frequent in patients with PD receiving dopaminergic replacement therapy. This cross-sectional study showed that $31.33 \%$ and $15.06 \%$ of Chinese PD patients experienced symptoms of motor fluctuations and dyskinesia. Compared with those reported in other populations, the prevalence of motor complications was relatively lower, especially dyskinesia. The reported rate of motor complications of PD patients showed a wide range, from $3 \%$ to $94 \%$, mostly $30 \%-74 \%[1-5,8]$. Motor complications usually occurred after 4-6 years of levodopa treatment [2]. In a Japanese investigation of 1768 PD patients, the incidence of patients who developed wearing-off was $21.3 \%, 59.4 \%$, and $73.2 \%$ at the end of the $5^{\text {th }}, 10^{\text {th }}$, and $15^{\text {th }}$ year after disease onset [4]. In a Chinese multicenter registry survey (excluding patients in Northwest of China), the incidence of wearing-off and dyskinesia was $46.5 \%$ and 
TABLE 4: The results of motor fluctuations and dyskinesia in different clinical stages.

\begin{tabular}{|c|c|c|c|c|c|c|c|}
\hline & Total $(n=166)$ & Motor fluctuations $(n=52)$ & $x^{2}$ & $P$ value & Dyskinesia $(n=25)$ & $x^{2}$ & $P$ value \\
\hline $\begin{array}{l}\text { Age of onset }(n, \%) \\
\leq 50 \text { years } \\
51-70 \text { years } \\
\geq 70 \text { years }\end{array}$ & $\begin{array}{c}29(17.47) \\
107(64.46) \\
30(18.07) \\
\end{array}$ & $\begin{array}{cc}18 & (62.07) \\
29 & (27.10) \\
5 & (16.67) \\
\end{array}$ & 16.625 & $<0.001$ & $\begin{array}{c}9(31.03) \\
15(14.02) \\
1(3.33)\end{array}$ & 9.101 & 0.011 \\
\hline $\begin{array}{l}\text { Duration of diseases } \\
<5 \text { years } \\
5-10 \text { years } \\
\geq 10 \text { years } \\
\end{array}$ & $\begin{array}{c}91(54.82) \\
59(35.54) \\
16(9.64) \\
\end{array}$ & $\begin{array}{ll}11 & (12.09) \\
28 & (47.46) \\
13 & (81.25) \\
\end{array}$ & 40.806 & $<0.001$ & $\begin{aligned} & 2(2.20) \\
& 12(20.34) \\
& 11(68.75) \\
&\end{aligned}$ & 48.692 & $<0.001$ \\
\hline $\begin{array}{l}\text { Duration of L-dopa treatment }(n, \%) \\
\leq 2 \text { years } \\
2-5 \text { years } \\
5-10 \text { years } \\
\geq 10 \text { years }\end{array}$ & $\begin{array}{c}98(59.04) \\
36(21.69) \\
23(13.86) \\
9(5.41) \\
\end{array}$ & $\begin{aligned} & 7(7.12) \\
& 21(58.33) \\
& 17(73.91) \\
& 7(77.78) \\
&\end{aligned}$ & 67.265 & $<0.001$ & $\begin{aligned} & 0(0.00) \\
& 11(30.56) \\
& 9(39.13) \\
& 5(55.56) \\
&\end{aligned}$ & 46.087 & $<0.001$ \\
\hline $\begin{array}{l}\text { Modified Hoehn-Yahr stages }(n, \%) \\
\begin{array}{l}1-1.5 \\
2-2.5 \\
3-4\end{array}\end{array}$ & $\begin{array}{ll}51(30.72) \\
76(45.78) \\
39(23.50) \\
\end{array}$ & $\begin{array}{c}6(11.76) \\
21(27.63) \\
25(64.10) \\
\end{array}$ & 29.030 & $<0.001$ & $\begin{array}{c}0(0.00) \\
6(7.89) \\
19(48.72) \\
\end{array}$ & 46.030 & $<0.001$ \\
\hline $\begin{array}{l}\text { Daily levodopa dosages }(n, \%) \\
\quad \leq 300 \mathrm{mg} \\
\quad 300-600 \mathrm{mg} \\
\geq 600 \mathrm{mg}\end{array}$ & $\begin{array}{l}49(29.52) \\
91(54.82) \\
26(15.66)\end{array}$ & $\begin{array}{l}6(12.24) \\
31(34.07) \\
15(57.69)\end{array}$ & 17.012 & $<0.001$ & $\begin{array}{c}4(8.16) \\
13(14.29) \\
8(30.77)\end{array}$ & 6.880 & 0.032 \\
\hline
\end{tabular}

TABLE 5: Results of logistic regression analysis in patients with motor fluctuations and dyskinesias.

\begin{tabular}{|c|c|c|c|c|c|c|}
\hline & & $B$ & Walds, $\chi^{2}$ & OR & 95\% CI & $P$ value \\
\hline \multirow{4}{*}{ Motor fluctuations } & Duration of levodopa therapy & 0.029 & 19.754 & 1.030 & $1.016 \sim 1.043$ & $<0.001$ \\
\hline & Bradykinesia-rigidity type & 1.115 & 5.417 & 3.051 & $1.164 \sim 7.997$ & 0.023 \\
\hline & Age of onset & -0.070 & 7.677 & 0.933 & $0.888 \sim 0.980$ & 0.006 \\
\hline & Constant & 2.884 & 3.492 & 17.885 & & 0.062 \\
\hline \multirow{3}{*}{ Dyskinesias } & Duration of disease & 0.307 & 14.653 & 1.359 & $1.161 \sim 1.590$ & $<0.001$ \\
\hline & Bradykinesia-rigidity type & -2.100 & 7.750 & 8.170 & $1.862 \sim 35.849$ & 0.005 \\
\hline & Constant & -4.065 & 27.618 & 0.017 & & $<0.001$ \\
\hline
\end{tabular}

Abbreviations: B: beta, OR: odds ratio, and CI: confidence interval.

$10.3 \%$, respectively [5]. The results of this survey, which used the wearing-off questionnaire of nine symptoms (WOQ-9) to defined wearing-off, were similar to ours.

PD with early onset often develops dyskinesia, which may occur in the first two years of levodopa treatment, especially in patients who develop disease before age 50 [14]. In the present study, the prevalence of motor fluctuations in PD patients with disease onset at $<50,50-70$, and after 70 years of age was $62.07 \%, 27.10 \%$, and $16.67 \%$, respectively. The prevalence of dyskinesia was $31.03 \%$, $14.02 \%$, and $3.33 \%$, respectively. Ferguson et al. found that autopsy confirmed cases of early onset to PD (age at onset under 50 years) that had a higher cumulative incidence of dyskinesia, wearing-off, and on-off [15]. A 59-month follow-up cohort study showed that the younger age at onset was an independent risk factor of development of motor fluctuations [16]. These results suggested that it is appropriate to decide when to use levodopa therapy according to the age of onset.

In the present study, bradykinesia-rigidity dominant phenotype was an independent risk factor for motor fluctuation and dyskinesia. Previous observational studies showed that patient who presented tremor-dominant subtype as the initial manifestation was less likely to develop dyskinesia $[17,18]$. This result was correlated with pathophysiological mechanisms of different clinical type. The tremor-dominant type is characterized by more severe cell impairment in the medial substantia nigra (SN) and more hyperactive in thalamo-motor and cerebellar projections. However, bradykinesia-rigidity dominant type, ventrolateral part of $\mathrm{SN}$, and posterior putamen showed more severe cell loss, in which the glutamatergic thalamocortical pathway was inhibited, and cortical activation was reduced [19]. More recent studies showed that tremordominant PD patients had higher level of $\alpha$-synucleinopathy and amyloid- $\beta$ markers in cerebrospinal fluid than that in postural instability and gait difficulties phenotype [20].

Based on the logistic analysis, we found that the duration of levodopa treatment was significantly associated with the motor fluctuations. On the contrary, the duration of disease may be a risk factor of dyskinesia. Previous studies showed that the duration of levodopa treatment and disease duration were independent risk factors in generating wearing-off and dyskinesia [21, 22]. A study on Chinese PD patients found that duration of levodopa treatment was the best predictor of 
motor fluctuations, while disease duration was the best predictor for dyskinesias among the risk factors of motor complications [23]. The pathophysiology of wearing-off is multifactorial, involving both the loss of presynaptic dopamine neuronal and dopaminergic storage capacity, particularly in the anterior putamen and postsynaptic mechanisms. Dopamine receptor supersensitivity is thought to contribute to the development of dyskinesia. The buffering capacity of dopamine transporter is lost, and the short half-life of levodopa and pulsatile release of dopamine are considered to be the major risk factors for developing levodopa-induced motor complications. What's more, the overexpression and phosphorylation of glutamate system of $\mathrm{N}$-methyl-D-aspartate (NMDA) receptors appear to be associated with the development of levodopa-induced dyskinesia [24]. Levodopa-induced dyskinesia has been found to be associated with genetic polimorphisms, such as the brainderived neurotrophic factor (BDNF), DRD3G3127A, and DRD2/ANKK1 gene region $[25,26]$. Some studies showed that levodopa may induce motor fluctuations and dyskinesia by dose-dependent manner. A study of sub-Sahara PD population comparing with matched Italian patients showed that motor fluctuations and dyskinesia were not associated with the duration of levodopa therapy, but with longer disease duration and higher levodopa daily dose [27].

A previous research has shown a significant and actual linear relationship between levodopa dose and dyskinesia [28]. The STRIDE-PD trial concluded that at the daily dosage of levodopa less than $400 \mathrm{mg}$ may reduce the occurrence of motor complications [29]. A Chinese study showed that a safe levodopa daily dosage was not more than $275 \mathrm{mg}$ or $4.2 \mathrm{mg} / \mathrm{kg}$ [21]. Cumulative levodopa dosage, which included both levodopa treatment duration and levodopa dosage, was another predictive of wearing-off [30]. In order to delay the onset of motor complications, some studies demonstrated that dopamine agonists in place of levodopa was first-line therapy [31]. In the present study, the main dopamine agonists used pramipexole and piribedil, and the proportions of these two dopamine agonists were higher in patients with motor complications compared to those without motor complications. Some studies have showed good efficacy of dopamine agonists in improving motor symptoms, and dopamine agonists can delay dyskinesia [32-34].

In conclusion, although levodopa remains the most effective treatment for PD and its use is related to better outcomes, for some patients with high risk of levodopainduced motor complications, delaying the use of levodopa and reducing the dose of levodopa may be a better clinical choice. For patients with early stage, especially bradykinesiarigidity type patients, dopamine agonists and MAO-B inhibitors may provide benefit when motor symptoms are not disabling and do not cause functional impairment in activities of daily life.

\section{Data Availability}

The data used to support the findings of this study are included within the article.

\section{Conflicts of Interest}

The authors declare that they have no conflicts of interest.

\section{Authors' Contributions}

The study was designed and organized by Dr. Jin Qiao. The data collection works were completed by the study group including Baihua Sun, Tao Wang, and Nianying Li. Finally, Baihua Sun finished the statistical analysis and article writing. Dr. Jin Qiao supervised the study and revised the manuscript.

\section{Acknowledgments}

This study was funded by the Science and Technology Department of Shaanxi Province, China (No. 2018SF-016).

\section{References}

[1] A. J. Espay, F. Morgante, A. Merola et al., "Levodopa-induced dyskinesia in parkinson disease: current and evolving concepts," Annals of Neurology, vol. 84, no. 6, pp. 797-811, 2018.

[2] T. N. Tran, T. N. N. Vo, K. Frei, and D. D. Truong, "Levodopainduced dyskinesia: clinical features, incidence, and risk factors," Journal of Neural Transmission, vol. 125, no. 8, pp. 1109-1117, 2018.

[3] F. Stocchi, A. Antonini, P. Barone et al., "Early detection of wearing off in parkinson disease: the deep study," Parkinsonism \& Related Disorders, vol. 20, no. 2, pp. 204-211, 2014.

[4] S. Ouma, J. Fukae, S. Fujioka et al., "The risk factors for the wearing-off phenomenon in parkinson's disease in Japan: a cross-sectional, multicenter study," Internal Medicine, vol. 56, no. 15, pp. 1961-1966, 2017.

[5] W. Chen, Q. Xiao, M. Shao et al., "Prevalence of wearing-off and dyskinesia among the patients with parkinson's disease on levodopa therapy: a multi-center registry survey in mainland China," Translational Neurodegeneration, vol. 3, no. 1, p. 26, 2014.

[6] A. Manson, P. Stirpe, and A. Schrag, "Levodopa-induceddyskinesias clinical features, incidence, risk factors, management and impact on quality of life," Journal of Parkinson's Disease, vol. 2, no. 3, pp. 189-198, 2012.

[7] R. B. Postuma, D. Berg, M. Stern et al., "MDS clinical diagnostic criteria for parkinson's disease," Movement Disorders, vol. 30, no. 12, pp. 1591-1601, 2015.

[8] R. Stowe, S. Patel, C. Rick, R. Gray, and C. E. Clarke, "Systematic review of levodopa dose equivalency reporting in parkinson's disease," Movement Disorders, vol. 25, no. 15, pp. 2649-2653, 2010.

[9] R. Bhidayasiri, N. Hattori, B. Jeon et al., "Asian perspectives on the recognition and management of levodopa "wearingoff" in parkinson's disease," Expert Review of Neurotherapeutics, vol. 15, no. 11, pp. 1285-1297, 2015.

[10] M. Stacy, A. Bowron, M. Guttman et al., "Identification of motor and nonmotor wearing-off in parkinson's disease: comparison of a patient questionnaire versus a clinician assessment," Movement Disorders, vol. 20, no. 6, pp. 726-733, 2005.

[11] K. L. Chou, M. Stacy, T. Simuni et al., "The spectrum of "off” in parkinson's disease: what have we learned over 40 years?" Parkinsonism \& Related Disorders, vol. 51, pp. 9-16, 2018. 
[12] C. C. Aquino and S. H. Fox, "Clinical spectrum of levodopainduced complications," Movement Disorders, vol. 30, no. 1, pp. 80-89, 2015.

[13] N. Giladi and A. Nieuwboer, "Medical treatment of freezing of gait," Movement Disorders, vol. 23, no. 2, pp. S482-S488, 2008.

[14] S. Ku and G. A. Glass, "Age of parkinson's disease onset as a predictor for the development of dyskinesia," Movement Disorders, vol. 25, no. 9, pp. 1177-1182, 2010.

[15] L. W. Ferguson, A. H. Rajput, and A. Rajput, "Early-onset vs. late-onset parkinson's disease: a clinical-pathological study," Canadian Journal of Neurological Sciences/Journal Canadien des Sciences Neurologiques, vol. 43, no. 1, pp. 113-119, 2016.

[16] N. W. Scott, A. D. Macleod, and C. E. Counsell, "Motor complications in an incident parkinson's disease cohort," European Journal of Neurology, vol. 23, no. 2, pp. 304-312, 2016.

[17] S. Kipfer, M. A. Stephan, W. M. Schupbach, P. Ballinari, and A. Kaelin-Lang, "Resting tremor in parkinson disease," Archives of Neurology, vol. 68, no. 8, pp. 1037-1039, 2011.

[18] Y.-H. Zhang, B.-S. Tang, C.-Y. Song et al., "The relationship between the phenotype of parkinson's disease and levodopainduced dyskinesia," Neuroscience Letters, vol. 556, pp. 109112, 2013.

[19] K. A. Jellinger, "Recent developments in the pathology of parkinson's disease," Ageing and Dementia Current and Future Concepts, vol. 62, pp. 347-384, 2002.

[20] G. Alves, K. F. Pedersen, B. R. Bloem et al., "Cerebrospinal fluid amyloid- and phenotypic heterogeneity in de novo parkinson's disease," Journal of Neurology, Neurosurgery \& Psychiatry, vol. 84, no. 5, pp. 537-543, 2013.

[21] H. Chen, J. Fang, F. Li, L. Gao, and T. Feng, "Risk factors and safe dosage of levodopa for wearing-off phenomenon in Chinese patients with parkinson's disease," Neurological Sciences, vol. 36, no. 7, pp. 1217-1223, 2015.

[22] C. Juri Clavería, C. Aguirre M, P. Viviani G, and P. Chaná Cuevas, "Factores de riesgo asociados con el desarrollo de complicaciones motoras en la enfermedad de parkinson: experiencia en población chilena," Revista de Neurología, vol. 45, no. 2, pp. 77-80, 2007.

[23] W. F. Kum, J. Gao, S. S. K. Durairajan et al., "Risk factors in development of motor complications in Chinese patients with idiopathic parkinson's disease," Journal of Clinical Neuroscience, vol. 16, no. 8, pp. 1034-1037, 2009.

[24] I. Ahmed, S. K. Bose, N. Pavese et al., "Glutamate NMDA receptor dysregulation in parkinson's disease with dyskinesias," Brain: A Journal of Neurology, vol. 134, no. 4, pp. 979-986, 2011.

[25] C. Comi, M. Ferrari, F. Marino et al., "Polymorphisms of dopamine receptor genes and risk of 1-dopa-induced dyskinesia in parkinson's disease," International Journal of Molecular Sciences, vol. 18, no. 2, p. 242, 2017.

[26] M. Rieck, A. F. Schumacher-Schuh, V. Altmann et al., "DRD2haplotype is associated with dyskinesia induced by levodopa therapy in parkinson's disease patients," Pharmacogenomics, vol. 13, no. 15, pp. 1701-1710, 2012.

[27] R. Cilia, A. Akpalu, F. S. Sarfo et al., "The modern prelevodopa era of parkinson's disease: insights into motor complications from sub-saharan Africa," Brain: A Journal of Neurology, vol. 137, no. 10, pp. 2731-2742, 2014.

[28] M. Chondrogiorgi, A. Tatsioni, H. Reichmann, and S. Konitsiotis, "Dopamine agonist monotherapy in parkinson's disease and potential risk factors for dyskinesia: a metaanalysis of levodopa-controlled trials," European Journal of Neurology, vol. 21, no. 3, pp. 433-440, 2014.
[29] C. Warren Olanow, K. Kieburtz, O. Rascol et al., "Factors predictive of the development of levodopa-induced dyskinesia and wearing-off in parkinson's disease," Movement Disorders, vol. 28, no. 8, pp. 1064-1071, 2013.

[30] R. A. Hauser, M. P. McDermott, and S. Messing, "Factors associated with the development of motor fluctuations and dyskinesias in parkinson disease," Archives of Neurology, vol. 63 , no. 12 , pp. $1756-1760,2006$.

[31] C. W. Olanow and J. A. Obeso, "Preventing levodopa-induced dyskinesias," Annals of Neurology, vol. 47, no. 1, pp. S167S168, 2000.

[32] Z. Zhang, J. Wang, X. Zhang et al., “An open-label extension study to evaluate the safety of ropinirole prolonged release in Chinese patients with advanced parkinson's disease," Current Medical Research and Opinion, vol. 31, no. 4, pp. 723-730, 2015.

[33] M. Takanashi, Y. Shimo, T. Hatano, G. Oyama, and N. Hattori, "Efficacy and safety of a once-daily extendedrelease formulation of pramipexole switched from an immediate-release formulation in patients with advanced parkinson's disease: results from an open-label study," Drug Research, vol. 63, no. 12, pp. 639-643, 2013.

[34] R. Constantinescu, M. Romer, M. P. McDermott, C. Kamp, and K. Kieburtz, "Impact of pramipexole on the onset of levodopa-related dyskinesias," Movement Disorders, vol. 22, no. 9, pp. 1317-1319, 2007. 